\title{
Structure and variation of CRISPR and CRISPR-flanking regions in deleted-direct repeat region Mycobacterium tuberculosis complex strains
}

Paul Jeffrey Freidlin ${ }^{1 *}$, Israel Nissan ${ }^{1}$, Anna Luria ${ }^{1,2}$, Drora Goldblatt ${ }^{1}$, Lana Schaffer ${ }^{3}$, Hasia Kaidar-Shwartz ${ }^{1}$ Daniel Chemtob ${ }^{4}$, Zeev Dveyrin ${ }^{5}$, Steven Robert Head ${ }^{3}$ and Efrat Rorman ${ }^{5}$

\begin{abstract}
Background: CRISPR and CRISPR-flanking genomic regions are important for molecular epidemiology of Mycobacterium tuberculosis complex (MTBC) strains, and potentially for adaptive immunity to phage and plasmid DNA, and endogenous roles in the bacterium. Genotyping in the Israel National Mycobacterium Reference Center Tel-Aviv of over 1500 MTBC strains from 2008-2013 showed three strains with validated negative 43-spacer spoligotypes, that is, with putatively deleted direct repeat regions (deleted-DR/CRISPR regions). Two isolates of each of three negative spoligotype MTBC (a total of 6 isolates) were subjected to Next Generation Sequencing (NGS). As positive controls, NGS was performed for three intact-DR isolates belonging to T3_Eth, the largest multiple-drug-resistant (MDR)-containing African-origin cluster in Israel. Other controls consisted of NGS reads and complete whole genome sequences from GenBank for 20 intact-DR MTBC and for 1 deleted-DR MTBC strain recognized as CAS by its defining RD deletion.
\end{abstract}

Results: NGS reads from negative spoligotype MTBC mapped to reference H37Rv NC_000962.3 suggested that the DR/CRISPR regions were completely deleted except for retention of the middle IS6110 mobile element. Clonally specific deletion of CRISPR-flanking genes also was observed, including deletion of at least cas2 and cas 1 genes. Genomic RD deletions defined lineages corresponding to the major spoligotype families Beijing, EAl, and Haarlem, consistent with 24 loci MIRU-VNTR profiles. Analysis of NGS reads, and analysis of contigs obtained by manual PCR confirmed that all 43 gold standard DR/CRISPR spacers were missing in the deleted-DR genomes.

Conclusions: Although many negative spoligotype strains are recorded as spoligotype-international-type (SIT) 2669 in the SITVIT international database, this is the first time to our knowledge that it has been shown that negative spoligotype strains are found in at least 4 different 24 loci MIRU-VNTR and RD deletion families. We report for the first time negative spoligotype-associated total loss of CRISPR region spacers and repeats, with accompanying clonally specific loss of flanking genes, including at least CRISPR-associated genes cas2 and cas 1. Since cas 1 deleted E.coli shows increased sensitivity to DNA damage and impaired chromosomal segregation, we discussed the possibility of a similar phenotype in the deleted-DR strains and Beijing family strains as both lack the cas 1 gene.

Keywords: Mycobacterium tuberculosis complex MTBC, Next Generation Sequencing NGS, CRISPR-Cas, Spacers, cas 1, IS6110, Deleted-direct repeat region deleted-DR, Region of difference deletion RD deletion, Spoligotype, MIRU-VNTR

\footnotetext{
* Correspondence: paul.freidlin@phlta.health.gov.il

'National Mycobacterium Reference Center, National Public Health

Laboratory Tel Aviv, Ministry of Health, Tel Aviv, Israel

Full list of author information is available at the end of the article
} 


\section{Background}

The National Public Health Laboratory Tel Aviv, National Mycobacterium Reference Center routinely genotypes all new culture-positive tuberculosis cases in Israel [1] with 43 spacers reverse line blot spacer oligonucleotide typing (spoligotyping) [2-4], and 24 loci mycobacterial interspersed repeat units - variable number tandem repeats (MIRU-VNTR) typing [5] using multiplex PCR and capillary electrophoresis $[6,7]$. The results are stored in Excel (Microsoft), BioNumerics (Applied Maths, Belgium), and WHOnet databases. From 2008-2013, over 1500 strains were genotyped and tested for drug sensitivity to first line, and when necessary, second line drugs. Six drug-sensitive isolates from three different patients $\mathrm{A}, \mathrm{B}$, and $\mathrm{C}$ (two isolates per patient) yielded negative spoligotyping results and three different 24 loci MIRU-VNTR profiles respectively (Additional file 1: Table S1).

Variation in the structure of CRISPR and CRISPRflanking regions of these negative spoligotype MTBC isolates was observed when NGS reads were mapped on reference whole genome sequence H37Rv NC_000962.3. This yielded visualization of coverage (Fig. 1). After substitution of manual-PCR-derived DR-spanning sequences for the corresponding map-derived regions, further details of structural variation were revealed by alignments of annotated subsequences containing CRISPR plus CRISPR-flanking regions to the annotated reference H37Rv sequence (Fig. 2).

In addition to the 6 drug-sensitive deleted-DR isolates, two drug sensitive and 1 multiple-drug-resistant (MDR) intact-DR strains from T3_Eth (SIT 149), our largest African-origin MDR-containing cluster, were analyzed by NGS (Table 1, Additional file 1: Table S1, Additional file 2: List S1). These provided positive controls for the sensitivity of our methods to correctly characterize from NGS data, the CRISPR-Cas regions of intact-DR $\mathrm{MTBC}$, especially with respect to the presence or absence of CRISPR spacers.

Complete loss of all Direct Repeat region (DR) repeats and spacers, negative spoligotyping results, and loss of genes in flanking regions has been reported previously for MTBC with negative spoligotypes [8,9] identical to those currently assigned to SIT 2669 [10] and for other bacteria [11], however, the molecular structures of the CRISPR regions (the newer name for DR) and flanking CRISPR-associated (Cas) regions [12] were not reported, except for a comparison to the homologous genomic region in non-tuberculous mycobacteria (NTM) [13]. The evidence reported herein clearly showed that the deleted-DR and accompanying deleted CRISPR-flanking

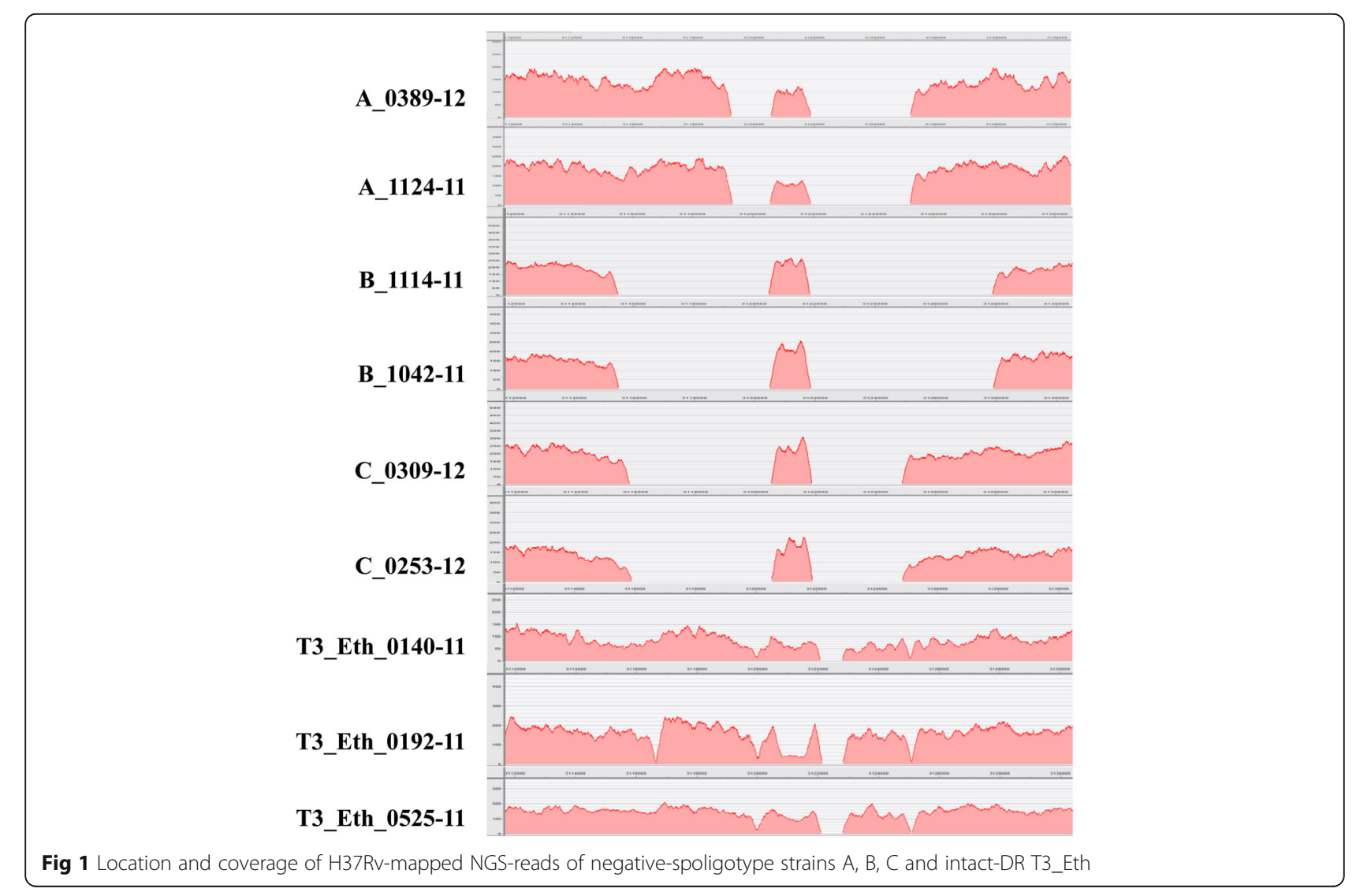




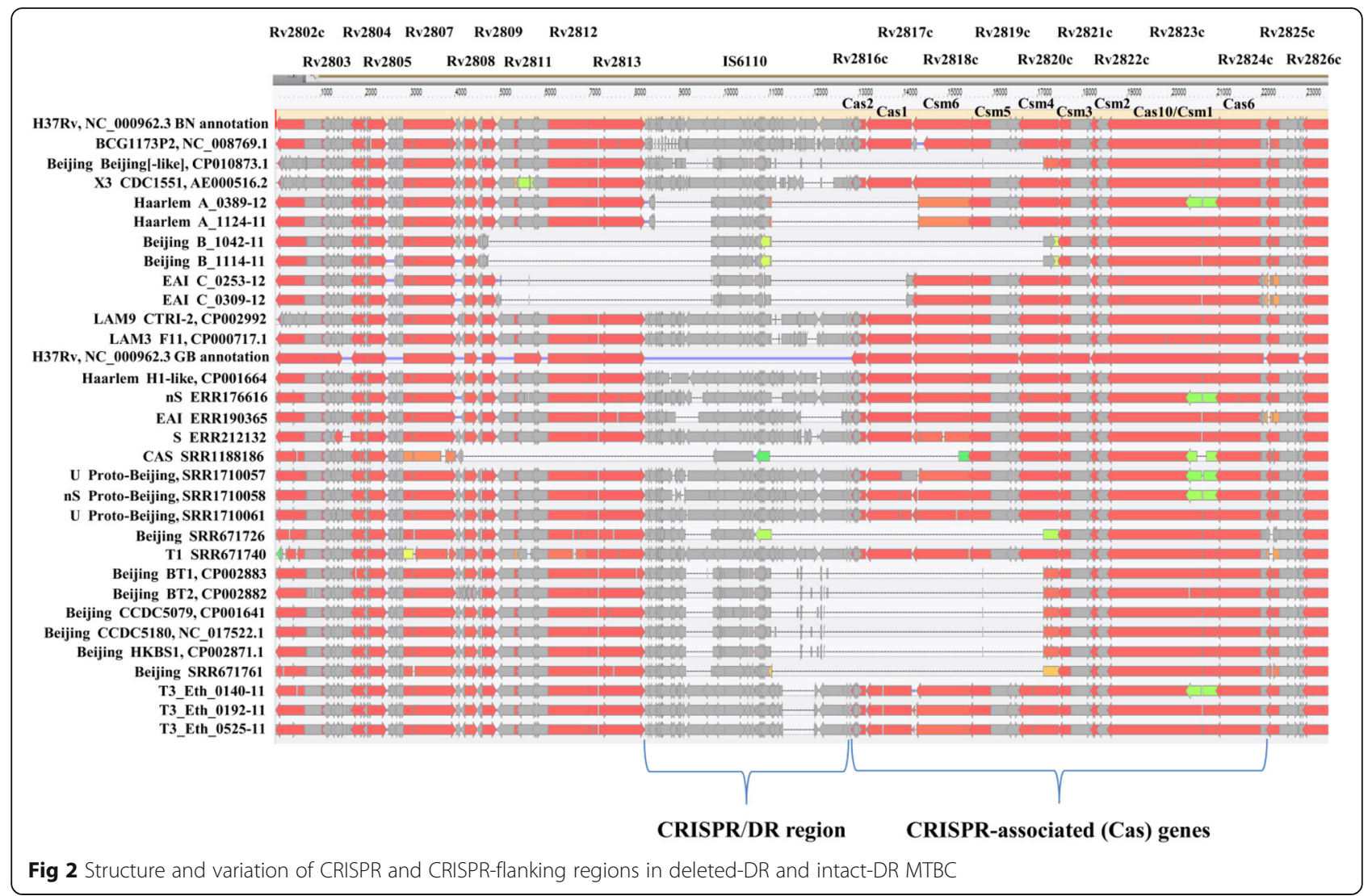

genes of the MTBC described in this study were not an instance of the ORFs absent in NTM species (Fig. 2). Also, this study is the first known to us to employ identification of Region of Difference (RD) deletions in order to assign negative spoligotype MTBC to their corresponding major spoligotype families.

The MTBC RD deletions are genomic long sequence polymorphisms (LSPs) [14, 15] which were detected in this study by aligning Mycobacterium tuberculosis complete genome sequences (obtained from public online databases or derived from NGS reads mapped on the H37Rv genome, Table 1) with the MTBC reference strain $\mathrm{H} 37 \mathrm{Rv}$ complete genome sequence [16]. The MTBC RD deletions, and also an extensive set of single nucleotide polymorphisms (SNPs), have been shown by Coll et al. [17] in an on-line available application for analysis of NGS reads, to have phylogenetic behavior that corresponds well with the phylogenetic distribution obtained by analysis of 43-spacer spoligotypes. 43-spacer spoligotypes have been assigned type numbers and family names $[10,18]$. The 43-spacer spoligotypes are more informative than the RD types and SNP types, thus any given specific 43-spacer spoligotype cannot be inferred from an RD type or SNP type, but the spoligotype family can be inferred from RD or SNP information, and in addition some sub-lineage information can be obtained from the SNP type [17]. Therefore, when one encounters a negative spoligotype by conventional screening for the 43 CRISPR spacers, if NGS sequencing can be performed for the deleted-DR strain, two convenient ways exist to determine the spoligotype family: 1) map the NGS reads to reference $\mathrm{H} 37 \mathrm{Rv}$, align the constructed MTBC sequence with the H37Rv sequence, and search for the relevant RD deletions, and 2) submit the NGS reads to the Coll et al. [17] site for analysis of RD deletions and SNPs. The RD data shown in Table 1 suggested that a third way could be developed which would not require sequencing for NGS reads: a possible multiplex PCR probe for relevant RD deletions, which could be analyzed by fragment analysis as in MIRU-VNTR typing, or by real time PCR. Various methods have been proposed for spoligotyping from NGS reads [19-22], but all involve detection of CRISPR spacers and thus would only yield negative results with our deleted-DR negative spoligotype strains.

A combination of the PCR-based methods 43 spacer spoligotyping and 24 loci MIRU-VNTR typing has replaced RFLP-typing as the MTBC genotyping screen of choice, due to the speed and convenience of the PCRbased methods, genotype discrimination as good as or better than that for RFLP-typing, and the ability to store the PCR-based results in easily manipulated digital format [5]. On a population level, screening for spoligotype 
Table 1 Characteristics of NGS sequenced deleted-DR sample strains A, B, C and intact-DR T3_Eth, and whole genome sequences obtained on-line or assembled from on-line read sets\#

\begin{tabular}{|c|c|c|c|c|c|c|c|c|c|c|c|c|c|c|c|}
\hline \multirow[t]{2}{*}{ MTBC Strain, ID } & \multirow[t]{2}{*}{ Spoligotype Octal } & \multirow{2}{*}{$\begin{array}{l}\text { SIT or RD } \\
\text { Family }\end{array}$} & \multirow{2}{*}{$\begin{array}{l}\text { Drug } \\
\text { Res }\end{array}$} & \multirow{2}{*}{$\begin{array}{l}\text { CRISPR } \\
\text { IS6110 }\end{array}$} & \multicolumn{3}{|c|}{$\underline{\text { mutation in }}$} & \multicolumn{8}{|c|}{ regions of difference (RD) } \\
\hline & & & & & mutT4 & $T 2$ & $\overline{\text { ogt }}$ & 105 & 207 & 181 & 150 & 142 & 182 & 239 & 750 \\
\hline \multicolumn{16}{|l|}{ Beijing } \\
\hline BT1, CP002883 & 000000000003771 & 1,Beijing & $\mathrm{TDR}^{\mathrm{a}}$ & inv & 48 & 58 & 12 & del & del & del & wt & wt & wt & wt & wt \\
\hline BT2, CP002882 & 000000000003771 & 1,Beijing & $\mathrm{TDR}^{\mathrm{a}}$ & inv & 48 & 58 & 12 & del & del & del & wt & wt & wt & wt & $w t$ \\
\hline CCDC5079, CP001641 & 000000000003771 & 1,Beijing & $\mathrm{DS}^{\mathrm{b}}$ & inv & 48 & 58 & 12 & del & del & del & wt & wt & wt & wt & $w t$ \\
\hline CCDC5180, NC_017522.1 & 000000000003771 & 1,Beijing & $\mathrm{MDR}^{\mathrm{b}}$ & inv & 48 & 58 & 12 & del & del & del & wt & wt & wt & wt & $w t$ \\
\hline HKBS1, CP002871.1 & 000000000003771 & 1,Beijing & $\mathrm{DS}^{\mathrm{a}}$ & inv & 48 & 58 & 12 & del & del & del & wt & wt & wt & wt & wt \\
\hline Beijing [-like], CP010873.1 & 000000000003731 & 190,Beijing & $\mathrm{MDR}^{c}$ & inv & 48 & 58 & 12 & del & del & del & wt & wt & wt & wt & wt \\
\hline SRR671761 & 000000000003771 & 1,Beijing & $?^{d}$ & unk & 48 & 58 & 12 & del & del & del & del & wt & wt & wt & o \\
\hline SRR671726 & 000000000003770 & 1,Beijing & $X D R^{d}$ & unk & 48 & 58 & 12 & del & del & del & wt & wt & wt & wt & wt \\
\hline B_1042-11 & 000000000000000 & nS,Beijing & $\mathrm{DS}^{\mathrm{e}}$ & wt & 48 & 58 & 12 & del & ext & del & wt & wt & wt & wt & wt \\
\hline B_1114-11 & 000000000000000 & nS,Beijing & $\mathrm{DS}^{\mathrm{e}}$ & wt & 48 & 58 & 12 & del & ext & del & wt & wt & wt & $\mathrm{vt}$ & wt \\
\hline \multicolumn{16}{|l|}{ Proto-Beijing } \\
\hline SRR1710057 & 777777777777731 & $246, U$ & $\operatorname{panS}^{d}$ & unk & wt & wt & wt & ext & wt & wt & wt & wt & wt & wt & $w t$ \\
\hline SRR1710058 & 777777777775331 & $\mathrm{nS}$ & panS $^{d}$ & unk & wt & wt & wt & ext & wt & wt & wt & wt & wt & wt & wt \\
\hline SRR1710061 & 777777777777771 & $523, \cup$ & $\operatorname{panS}^{d}$ & unk & wt & wt & wt & ext & wt & wt & wt & wt & wt & wt & wt \\
\hline \multicolumn{16}{|l|}{ Non-Beijing } \\
\hline ERR176616 & 777777607060771 & $\mathrm{nS}$ & $\mathrm{MDR}^{d}$ & unk & wt & wt & wt & wt & wt & wt & wt & wt & wt & wt & wt \\
\hline ERR190365 & 000037777403471 & nS,EAl & $D R^{d}$ & unk & wt & wt & wt & wt & wt & wt & wt & wt & wt & del & vo \\
\hline ERR212132 & 776337777760771 & $71, S$ & $\operatorname{panS}^{d}$ & unk & wt & wt & wt & wt & wt & wt & wt & wt & wt & wt & $w t$ \\
\hline SRR671740 & 777777777760771 & $53, \mathrm{~T} 1$ & $X D R^{d}$ & unk & wt & wt & wt & wt & wt & wt & wt & wt & wt & wt & wt \\
\hline SRR1188186 & 000000000000000 & $\mathrm{nS}, \mathrm{CAS}$ & $\operatorname{panS}^{d}$ & unk & wt & wt & wt & wt & new & wt & wt & wt & wt & wt & del \\
\hline A_0389-12 & 000000000000000 & nS,Haarlem & $\mathrm{DS}^{\mathrm{e}}$ & wt & wt & wt & 15 & wt & new & wt & wt & wt & del & wt & wt \\
\hline A_1124-11 & 000000000000000 & nS,Haarlem & $\mathrm{DS}^{\mathrm{e}}$ & wt & wt & wt & 15 & wt & new & wt & wt & wt & del & wt & $w t$ \\
\hline C_0253-12 & 000000000000000 & $\mathrm{nS}, \mathrm{EAl}$ & $\mathrm{DS}^{\mathrm{e}}$ & inv & wt & wt & wt & wt & new & wt & wt & wt & wt & del & $w t$ \\
\hline C_0309-12 & 000000000000000 & nS,EAl & $\mathrm{DS}^{\mathrm{e}}$ & inv & wt & wt & wt & wt & new & wt & wt & wt & wt & del & wt \\
\hline T3_Eth_0140-11 & 777000377760771 & 149,T3_Eth & $\mathrm{DS}^{\mathrm{e}}$ & unk & wt & wt & wt & wt & wt & wt & wt & wt & wt & wt & $w t$ \\
\hline T3_Eth_0192-11 & 777000377760771 & 149,T3_Eth & $\mathrm{MDR}^{\mathrm{e}}$ & unk & wt & wt & wt & wt & wt & wt & wt & wt & wt & wt & wt \\
\hline T3_Eth_0525-11 & 777000377760771 & 149,T3_Eth & $\mathrm{DS}^{\mathrm{e}}$ & unk & wt & wt & wt & wt & wt & wt & wt & wt & wt & wt & wt \\
\hline BCG1173P2, NC_008769.1 & 676773777777600 & $482, \mathrm{BCG}$ & $D R^{f}$ & wt & wt & wt & vt & wt & wt & wt & wt & del & wt & $\mathrm{vt}$ & wt \\
\hline CDC1551, AE000516.2 & 700076757760771 & $549, \times 3$ & $\mathrm{DS}^{\mathrm{g}}$ & wt & wt & wt & wt & wt & wt & wt & wt & wt & wt & wt & wt \\
\hline CTRI-2, CP002992 & 777777607760771 & 42,LAM9 & $\mathrm{DS}^{\mathrm{g}}$ & wt & wt & wt & wt & wt & wt & wt & wt & wt & wt & wt & wt \\
\hline F11, CP000717.1 & 776177607760771 & 33,LAM3 & $\mathrm{DS}^{\mathrm{g}}$ & wt & wt & wt & wt & wt & wt & wt & wt & wt & wt & wt & wt \\
\hline Haarlem, CP001664 & 775777774020751 & H1-like & $\mathrm{DR}^{\mathrm{g}}$ & wt & wt & wt & 15 & wt & wt & wt & wt & wt & del & wt & wt \\
\hline
\end{tabular}

"ID, sample, read set or NCBI accession number; octal, from spoligosome17, SIT, spoligotype international type and associated family; nS, no SIT; Drug Res, resistant to drug, I-isoniazide, R-rifampicin, E- ethanbutol, S-streptomycin, P-pyrazinamide, DS (drug sensitive): none, DR (drug resistant): at least one but not I\&R together: MDR (multiple drug resistant): at least I\&R, XDR (extensively drug resistant): at least MDR plus defined $2^{\text {nd }}$ line drugs consisting of at least 1 quinolone and at least one of the injectable drugs kanamycin, capreomycin or amikacin, TDR (totally drug resistant): resistant to all $1^{\text {st }}$ and $2^{\text {nd }}$ line drugs. a-g: Additional file 2: List S1 - sources of drug sensitivity data; wt, identical to reference genome H37Rv NC_000962.3; CRISPR IS6110, orientation of the IS6110 mobile element found in the direct repeat region, wt: same orientation as in H37Rv, inv: inverted, unk: unknown; mutation in mutT4 (Rv3908), codon 48: Arg CGG GGG Gly; mutation in mutT2 (Rv1 160), codon 58: Gly GGA CGA Arg; mutation in ogt (Rv1316c), silent codon 12: Gly CCC TCC Gly, missense codon 15: Thr GGT GCT Ser (note: ogt gene is in inverse orientation); regions of difference RD, 105: proto and all modern Beijing, 207: LSP in CRISPR region defining modern Beijing - includes cas gene deletions, 181-150-142: polymorphic LSPs for Beijing, 182: marker for Haarlem, 239: marker for EAl, 750: marker for CAS

family provides insight into the bio-geographic burden of $\mathrm{TB}$ in the world and for any particular country $[1,10,18]$. Thus it is useful to be able to determine the spoligotype family for a strain that has a negative spoligotype by conventional screening. While in general the more informative 24 loci MIRU-VNTR type will still phylogenetic-wise segregate according to its complementary spoligotype [5], for certain MIRU-VNTR 
profiles the miru-spoligo correlation may be difficult to establish. A published example of this [7] showed that the MIRU-VNTR profiles in spoligotype families CAS and Beijing can sometimes be difficult to distinguish. In another case, very similar MIRU-VNTR profiles were found in both the T and LAM spoligotype families (unpublished). In these cases it was not only useful, but indeed necessary, to determine at least the spoligotype family. On a clinical level, the combination of spoligotype and MIRU-VNTR type complements and guides classical epidemiological investigation to clarify questions of transmission between individuals, transmission chains among larger groups of individuals, questions about whether a recurrence of TB is due to reactivation or reinfection, and resolution of questions arising when a lab provides a positive TB report that is inconsistent with the physician's observation - that is- the possibility of a false positive due to lab contamination. Often 24 loci MIRU-VNTR alone supplementing classical epidemiology is enough to resolve these issues. Nevertheless, if the strains involved have negative spoligotypes on conventional screening, knowledge of at least the spoligotype family (and even better, the sub-lineage from SNP data) can help validate the results concluded from classical epidemiology supplemented with MIRUVNTR typing. Finally, there are often gaps in epidemiological knowledge when trying to establish transmission chains among larger groups of individuals. These knowledge gaps can be exacerbated by subtle polymorphisms (1 locus, 1 copy number) in MIRUVNTR profile, where knowing only the spoligotype family will not be enough to firmly establish the transmission chain. Therefore, when confronted with negative spoligotypes in large putative transmission chains, it seems reasonable to recommend that all the strains should be processed for NGS reads. Then the NGS reads for the negative spoligotype strains should be used for spoligotype family determination [17], and subsequently for SNP clonality-check comparisons among the strains to delineate the outbreak [23, 24].

Mycobacterium tuberculosis complex (MTBC) bacteria possess a Type III-A CRISPR-Cas system structurally homologous to Type III-A of other genera [12]. Active Type III-A systems provide adaptive immunity to bacteriophage and plasmids $[12,25,26]$, and perform endogenous roles, for example, endogenous DNA repair via Cas1 endonuclease [27].

In MTBC the CRISPR region is also known as the direct repeat region (DR). Polymorphism in the number and type of 43 spacers in the DR/CRISPR region of any given MTBC strain, has been and continues to be extensively used for molecular epidemiology of MTBC, especially for overviews of phylogenetic and bio-geographical distributions of clades $[2,4,10,18]$. Fourty three-spacer-
SPO-plus-24-loci-MIRU genotyping provides molecular epidemiology at the resolution of clonal transmission [5], with the exception of certain instances, for example, of convergent evolution [28].

The functional activity of the MTBC CRISPR-Cas system remains to be investigated, but the highly conserved sequence and order of the 43 CRISPR/DR region spacers [3] which is the basis for the epidemiological and phylogenetic usefulness of polymorphism on spacer oligonucleotide typing (spoligotyping), has been interpreted to imply at least partial inactivity of the MTBC CRISPRCas system [29]. This interpretation presumes that the insertion of new spacers in the CRISPR/DR region is one of the fundamentals of, and evidence for, adaptive immunity, and thus lack of evidence for insertion of new spacers in MTBC implies at least partial inactivation of the CRISPR-Cas adaptive immune system in MTBC. The MTBC Type III-A CRISPR-Cas system [12] is defined by the presence of cas10/csm1 and csm accessory genes, and is predicted to be in some manner active on the basis of the presence of intact cas 1 and cas 2 genes. In addition, some sort of activity even if only in endogenous roles, can be predicted from GenBank annotation that informs of mass spectroscopy support [30] for the existence of most of the cas gene products, including Cas1in the reference MTBC strain H37Rv. The lineagedefining RD 207 deletion [14, 31] of the super-spreader MDR-containing Beijing family includes complete deletion of cas1 and cas 2 genes (Fig. 2), so possession of a fully active Type III-A CRISPR-Cas system is not a prerequisite for a successful (with respect to pathogenicity, transmissibility, and adaptability) MTBC lineage. Interestingly, the complete loss of CRISPR spacers in E. coli gave the same DNA-repair deficient phenotype as deletion of the E. coli cas1 (ygbT) gene [27]. Using M. smegmatis as a model for MTBC [32], Cas2 [33] by itself was shown to be deleterious to Mycobacterium survival, thus it may be significant that deletion of cas 1 was always accompanied by deletion of at least cas 2 .

In summary, the results showed that complete deletion of DR region repeats and spacers in negative-spoligotype MTBC was accompanied by clonal-specific loss of flanking genes, always accompanied by complete deletion of cas2, complete deletion of putative (functional activity still unknown in MTBC) DNA-repair gene cas1, and retention of the middle IS6110 mobile element. For the first time it was shown that negative spoligotype MTBC nevertheless could be assigned to major spoligotype families by identification of NGS-revealed corresponding lineage-defining RD deletions. Since there was only 1 deleted-DR strain-type (based on at most two isolates) per RD-defined family, there was not enough information to decide if the loss of cas genes was solely clonally specific, or perhaps had lineage-defining features. 


\section{Methods}

Growth of Mycobacterium tuberculosis strains, and drug sensitivity tests

All approximately 1500 new culture-positive MTBC strains collected during 2008-2013 were analyzed. Cultures were grown and identified as MTBC as previously described [7]. Drug sensitivity tests were performed as previously described [7].

\section{Extraction of MTBC DNA}

Preparations of RFLP-grade MTBC DNA for NGS sequencing, and of "crude" sonicated, heated extract for genotyping, were done as previously described [7].

\section{Genotyping of MTBC DNA}

Routine genotyping of MTBC DNA by 43 spacer spoligotyping (reverse line blot kit, Omicum, India) and 24 loci MIRU-VNTR typing (in house multiplex PCR and amplicon-sizing by capillary electrophoresis) were done as previously described [6, 7]. BCGP2 was the generous gift of Professor Hillel Bercovier, Hebrew University of Jerusalem. European Communicable Disease Center Proficiency Test (ECDCPT) samples 21 and 25, and ECDCPT H37Rv control DNA were obtained originally as proficiency test materials [34]. Genotyping results were stored in an Excel (Microsoft) database, a BioNumerics ver7.6 database, and a WHONET database.

NGS Assay and bioinformatics analyses at the Scripps Research Institute, including 1) coverage-curves illustrating deletion of CRISPR/DR-Cas regions present in the template reference $\mathrm{H} 37 \mathrm{Rv}$ chromosome sequence, and 2) determination of spoligotypes from NGS reads

RFLP-grade DNA samples (micrograms of highly concentrated, highly purified, high quality DNA) were sent to Mgr. Steven Robert Head and Mgr. Lana Schaffer of the Scripps Research Institute in San Diego, California USA for NGS and draft bioinformatics analysis.

One microgram of each sample was sheared on the Covaris S2. Each sample was then end repaired, a-tailed and adapter ligated. The samples were cleaned with Agencourt beads and treated with 6 cycles of PCR. After PCR the samples were cleaned using Zymo 25 columns and loaded on a $2 \%$ agarose gel. The gel was cut from 400-700 bp and cleaned again with the Zymo25 column.

The samples were sequenced on an Illumina Miseq system as paired-end reads each with length $300 \mathrm{bp}$. The alignment was performed using Novoalign v3.00.05 (http://www.novocraft.com) without soft clipping to allow reads which cross the ends of the reference sequence as would be the case of a circular genome. The Mycobacterium tuberculosis reference sequence used for the alignment was MTB-H37Rv-asm19595v2 and for repeat sequences not in the mapped-to-H37Rv MTBC sequence was Mycobacterium bovis AF2122/97. The reads were preprocessed by trimming adaptor sequences (GATCGGAAGAGCACACGTCTG), trimming by base call quality scores lower than 26, removing unmapped reads and duplicate reads, and removal of reads with mapping quality score less than one. Repeat sequence coordinates and sequences were taken from the Jansen paper [35].

For coverage curves illustrating deletion of CRISPR-DRCas regions that were present in the template $\mathrm{H} 37 \mathrm{Rv}$ chromosome sequence, the aligned bam files were converted to wig files using bamtools, bedtools, and ucsctools. The wig files were imported into UCSC Microbial Genome Viewer (http://microbes.ucsc.edu).

Recovery of spoligotypes from NGS data was accomplished as follows. A bed file was created using the coordinates of the 43 DR repeat units. This bedfile and the aligned bam files were used with "samtools bedcov" tool to output number of counts for each repeat unit. In order to produce a binary table matching the spoligotyping regions with less than 500 counts were considered absent.

\section{Bioinformatics at the National Public Health Laboratory Tel Aviv}

Analyses of our NGS raw data were accomplished by use of BioNumerics version 7.5 and 7.6 (Applied Maths, Belgium) import and processing of 1) cleaned pairedend and single-end fastq reads received from Scripps, 2) reads formatted as sra (in fastq format) and fastq files downloaded from NCBI or other sources, and 3) fastq or $\mathrm{EMBO} / \mathrm{GB}$ format reference whole chromosome sequences or contributed chromosome sequences from NCBI or other sources. For visualization of the molecular details of the CRISPR-DR and flanking regions (including Cas genes), reads were resequenced/mapped onto reference H37Rv NC_000962.3 using default parameters with the following changes: accept pairedend reads, minimum coverage 10 , minimum sequence length before considered for contig $500 \mathrm{nt}$. The "coverage" was examined as percentage of the H37Rv genome matched, and as the number of reads contributing to each base pair assignment.

The mapped chromosomes were annotated with H37Rv GenBank and BioNumerics-predicted (based on possible reading frames) features and matrix compared and aligned in chromosome comparison mode using default parameters. For decisions about reading frames and codon assignments, reference H37Rv NC_000962.3 annotated only with GenBank annotation was included in comparisons.

Default parameters were: I. Comparison Project type: full sequence based; Matrix: matrix; self-comparison of sequences; direct sequence orientation; inverted sequence orientation; Seed extension DNA seed: minimum 
15 matches in window size of 25 bases; Amino acid seed: minimum 6 matches in window size of 10 amino acids; Stretch extension Minimum 15 matches in window size of 30 bases; Minimal stretch length: 50; II. Seeds Seed database: seed 11111, type: DNA, length:5; Seeds used in project: seed 11111, type: Transl, length:5, and III. Alignment settings template entry: usually the GB plus $\mathrm{BN}$ annotated reference $\mathrm{H} 37 \mathrm{Rv}$; stretch import minimum length: 0; Minimum identity: 0; Stretch mapping Open gap penalty: 10; Extend gap penalty 10; do NOT allow repetition of mapped stretches (box should NOT be checked); full alignment of stretch ends: checked; Full alignment settings: open gap penalty (\%): 100; extend gap penalty (1\%): 0 ; use fast algoritm: checked; K-tuple size:2; No. of diagonals (1-200): 99. This generated a schematic suitable for visualization of deletions, while choosing "show text view" while in alignment sub-mode in the comparison mode allowed visualization of single nucleotide polymorphisms (SNPs). An important point is that any given set of parameters has its own strengths and limitations. The default parameters of BioNumerics 7.5 and 7.6 Chromosome Comparison did not detect inverse IS6110 in the CRISPR-Cas region (it appeared as a deletion), nor were they suitable for comparison of shorter sequences (for example, sequences of 44 thousand nucleotides/nt or less).

The region containing the CRISPR-Cas sequence plus flanking regions was cut out (maximum approximately 44 thousand nt), annotated and used for further comparisons in the alignment window when high similarity was expected, or in the chromosome comparison mode and alignment sub-mode with appropriate parameters when some dissimilarity was expected.

The appropriate parameters for comparison of shorter sequences in the Chromosome Comparison mode were (only parameters differing from the default parameters detailed above are given): I. Comparison Seed extension: DNA seed: minimum 6 matches in window size of 15 bases; Amino acid seed: minimum two matches in window size of 5 amino acids; Stretch extension: minimum 6 matches in window size of 10 bases; Minimal stretch length: 12; II. Seeds seed database: seed: 111; type: DNA; length: 3; Seeds used in project: seed: 111; type: DNA; length: 3; III. Alignment settings: Full alignment settings: open gap penalty (\%): 0 ; No. of diagonals (1-200): 30 . Other parameter sets can be better for other uses, and must be determined empirically.

In order to obtain contig-corrected sequences for the six approximately $44 \mathrm{Kbp}$ CRISPR plus CRISPR-flanking subsequences of the deleted-CRISPR strains from patients $\mathrm{A}, \mathrm{B}$, and $\mathrm{C}$, the NGS-obtained H37Rv-mapped subsequences containing the CRISPR-spacers regions (the DR regions) were excised from the assembled mapped whole genome sequence at predetermined flanking genes on each side, and replaced with sequences of PCR validated contigs spanning the CRISPR-spacers-DR region, that is, with 5' and 3' ends of the contigs overlapping the conserved CRISPR-flanking genes (Fig. 2) characteristic for each deleted-DR strain. These "DRplus" sequences corrected for the appropriate contigs are found in Additional file 3: Sequences S1-S6. The CRISPR-flanking genes and their locations are listed in Additional file 4: Table S2.

The PCR validated contigs were constructed using primers inferred from the NGS data from mapped to $\mathrm{H} 37 \mathrm{Rv}$, and de novo, sequences (de novo sequences obtained using the open-source short-read assembly program "Ray" employed by the Power Assembler tool of Applied Mathematics software BioNumerics version 7.5 and version 7.6) according to which regions could be expected to be "conserved" in the CRISPR-spacers region itself (that is, the IS6110 mobile element), and in the immediately adjacent CRISPR-flanking regions characteristic for each deleted-DR strain. PCR and agarose gel electrophoresis were performed as previously described [7] and the PCR product purified on QIAQUICK (QIAGEN) columns. The purified PCR product was sent for forward and reverse sequencing to a commercial sequencing facility (HyLabs, Rehovot), and the sequences from the ab1 sequencing files were trimmed and used to construct contigs spanning the CRISPR-spacers region (BioNumerics version 7.5). Validation of sequence trimming and alignment for contig construction was done in parallel using a different bioinformatics tool (Clone Manager 9).

The H37Rv NC_000962.3 whole genome sequence locations, corresponding to the DR plus flanking regions (sequences named DRplus) of the mapped deleted-DR strains $\mathrm{A}, \mathrm{B}$, and $\mathrm{C}$, were used for alignments for primer construction (Additional file 4: Table S2). The major primers used for manual PCR were as shown in Additional file 5: Table S3.

Recovery of spoligotypes from NGS reads, or from downloaded reference or contributed chromosomes, was accomplished by constructing a new BioNumerics version 7.5 (now 7.6) application, manuscript in preparation. In addition, the sequences for the DR region-spanning contigs for deleted-DR strains A, B, and C were examined by NCBI BLAST for sequences corresponding to spacer sequences.

Drug sensitivity and lineage information for downloaded reads was obtained either from the literature (Table 1, Additional file 2: List S1) [14, 15] or by uploading the reads to TB Profiler [36] which reported back the drug sensitivities for first and second line drugs, and projected lineages. RD deletion confirmation of lineages (Table 1) was obtained from BioNumerics Chromosome Comparison alignments of complete genome sequences (downloaded whole, or derived from assembly of $\mathrm{H} 37 \mathrm{Rv}$ mapped-reads) to the reference $\mathrm{H} 37 \mathrm{Rv}$ sequence. 


\section{Results and Discussion}

Variation in the CRISPR and CRISPR-flanking regions: clonal specific deletion of CRISPR-flanking regions in deleted-DR strains - including deletion of at least cas 1 and cas2, and retention of the IS6110 mobile element in deleted-DR strains

In Fig. 1, coverage of the homologous reference H37Rv sequences was approximately 150 reads for each isolate, with the negative spoligotype isolates $\mathrm{A}, \mathrm{B}$, and $\mathrm{C}$ showing wide gaps to the left and right of the middle sequence. The middle read-coverage length was suggestive of that of an IS6110 mobile element. The coverage of the template H37Rv genome (NC_000962.3) (4411532 bp) ranged from 97.4 to $99.1 \%$ for the deleted-CRISPR-spacers-Cas strains A, B, and C, and 98.3 to $99.2 \%$ for the T3_Eth strains.

Variations in the CRISPR and CRISPR-flanking regions of negative spoligotype (Additional file 1: Table S1) MTBC isolates from patients $\mathrm{A}, \mathrm{B}$, and $\mathrm{C}$ were observed in the visualization of NGS reads mapped on reference H37Rv NC_000962.3 (Fig. 1) and annotated alignments of DRplus (DR plus flanking sequences) subsequences to the DRplus reference $\mathrm{H} 37 \mathrm{Rv}$ subsequence (Fig. 2). For comparison, intact-DR results from three NGS-sequenced T3_Eth were shown.

DRplus subsequences from isolates A, B, C, and isolate SRR1188186 which was assembled from downloaded reads, were aligned to reference template $\mathrm{H} 37 \mathrm{Rv}$ subsequence (Fig. 2, Additional file 4: Table S2). A, B, and $\mathrm{C}$ - specific (that is, clonally specific) deletions of CRISPR-flanking genes were observed, including deletions of cas genes to the right of the retained mid IS6110. All 4 deleted-DR strains, retained their mid IS6110 mobile element, and deleted at least cas 2 and cas1 (Fig. 2, Additional file 4: Table S2).

The deleted-DR $B$ isolates showed a right-side deletion of the same size and location as that shown in Beijingfamily strains (Fig. 2). Subsequent RD deletion identification of deleted-DR B isolates as Beijing (Table 1), suggested that the $\mathrm{B}$ deletion may be an extension of the Beijing lineage-defining RD207 deletion. This was further suggested by the fact that the deleted-DR $B$ isolates shared the Beijing-specific mutator phenotype SNPs mutT4|T2|ogt [37] (Table 1). Thus we propose to name the deletion seen in the deleted-DR B isolates as "extended RD207" in accordance with the terminology adopted by Luo et al. [31] for the RD105 deletion in proto-Beijing: extended RD105 (Table 1). It was interesting, but of as yet unproven important significance as discussed below, that in contrast to the inverse orientation of the IS6110 mobile element in the DR region of many if not all validated Beijing-family sequences (Additional file 6, Figure S1) the orientation of IS6110 in the Beijing deleted-DR B isolates was wild type, that is, the same as for reference template H37Rv (Additional file 6: Figure S1, Table 1).
Validation of retention of mid-IS6110 mobile element, and of loss of varying amounts of genomic regions flanking the deleted CRISPR/DR regions of isolates $A, B$, and $C$

In order to validate retention of IS6110, and clonally specific loss of flanking genomic sequences in deletedDR isolates $\mathrm{A}, \mathrm{B}$, and $\mathrm{C}$, primers were chosen from conserved flanking genes and the mid IS6110, and used to generate amplicons by manual PCR which were assembled into contigs for a DR-spanning sequence for each isolate $\mathrm{A}, \mathrm{B}$, and $\mathrm{C}$. These validated DR-spanning sequences were substituted into the DRplus sequences of $\mathrm{H} 37 \mathrm{Rv}$-mapped isolates $\mathrm{A}, \mathrm{B}$, and $\mathrm{C}$, resulting in a "corrected' DRplus sequence for each isolate $A, B$, and $C$ (Additional file 3: Sequences S1-6). Alignment of the corrected DRplus sequences to template H37Rv (Fig. 2) confirmed that mid-IS6110 was retained in each deletedDR isolate A, B, and C. It was interesting that the orientation of this IS6110 of each Beijing deleted-DR isolate B was wild type with respect to $\mathrm{H} 37 \mathrm{Rv}$, and inverse with respect to the orientation of IS6110 observed in reference whole genome Beijing sequences (Additional file 6: Figure S1, Table 1). Alignment of the manual PCR corrected DR plus flanking sequences to template H37Rv (Fig. 2) also confirmed the clonally specific loss of CRISPR-flanking regions, as shown in Fig. 2 and detailed in Additional file 4: Table S2. Each deleted-DR isolate $\mathrm{A}, \mathrm{B}$, and $\mathrm{C}$ showed deletion of at least cas 2 and cas1 from among the cas genes (Fig. 2, Table 2, Additional file 4: Table S2).

The absence of mutations in $\operatorname{csm} 3, \operatorname{csm} 2$, and cas6 (Table 2) could mean that those genes cannot tolerate mutations due to the importance of their sequence, or transcription or translation products. However, mutations could appear if a larger set of strains were examined, a possibility suggested by the recent report that the cas 6 gene is dispensable in Type III CRISPR-cas systems [12].

\section{Did the retained IS6110 mobile element have a role in generating the CRISPR and flanking-sequences deletions observed in the deleted-DR strains?}

The mobile element IS6110 inserts into the Mycobacterium tuberculosis genome in a variety of ways with a variety of potential effects [38], some of which we have discussed previously [7]. The DR/CRISPR region is a hot spot for IS6110 insertions, where there can be 0,1 , or more IS6110 elements inserted in the DR region [39, 40]. In this study all the MTBC strains examined had 1 mid DR IS6110 mobile element (Fig. 2). After CRISPR deletion, along with deletion of varying amounts of DR-flanking sequences, the DR region was left with a residual IS6110 mobile element attached to what remained of the flanking sequences (Fig. 2). The IS6110 mobile element has the ability to delete sequences on either side of it [39]. In the MTBC Beijing family, the mid DR IS6110 mobile 
Table 2 The presence or absence of mutations in CRISPR-associated genes in Mycobacterium tuberculosis complex strains compared to reference template H37Rv NC_000962.3 4411532 bp. The mutations are listed in ascending order of their location relative to the reference H37Rv chromosome

\begin{tabular}{|c|c|c|c|c|c|c|}
\hline Type & Gene & Location & SNP & Change & Description & Rv \\
\hline deletion & $\operatorname{cas} 2$ & 3123625 & & & Beijing RD207 \& all deleted-CRISPR strains & Rv2816c \\
\hline deletion & cas 1 & 3123967 & & & Beijing RD207 \& all deleted-CRISPR strains & Rv2817c \\
\hline SNP & cas 1 & 3124014 & $\mathrm{cga} / \mathrm{cgc}$ & Ser/S->Ala/A & T3_Eth defining & Rv2817c \\
\hline SNP & $\operatorname{cas} 1$ & 3124352 & gct/gat & Ser/S->lle/l & T3_Eth defining & Rv2817c \\
\hline SNP & $\operatorname{cas} 1$ & 3124743 & $\mathrm{cac} / \mathrm{caa}$ & Val/N->Leu/L & proto-Beijing defining & Rv2817c \\
\hline deletion & csm6 & 3124996 & & & Beijing RD207 \& deleted-DR A (Haarlem) & Rv2818c \\
\hline SSP & csm6 & 3125070 & & & T3_Eth specific & Rv2818c \\
\hline SSP\&SNPS & csm6 & 3125120 & & & proto-Beijing specific & Rv2818c \\
\hline SNP & csm6 & 3125171 & gtt/gct & Asn/N-> Ser/S & ERR212132 specific & Rv2818c \\
\hline SSP & csm6 & 3125690 & & & ERR212132 specific & Rv2818c \\
\hline SNP & csm6 & 3125235 & $\operatorname{ttg} / \operatorname{tta}$ & Gln/Q- > stop & BCG specific & Rv2818c \\
\hline deletion & csm5 & 3126240 & & & Beijing RD207 & Rv2819c \\
\hline SNP & $\operatorname{csm} 5$ & 3126279 & atc/att & Asp/D- > Asn/N & SRR671740 specific & Rv2819c \\
\hline SNP & csm5 & 3127273 & $\mathrm{gtg} / \mathrm{gtt}$ & $\mathrm{His} / \mathrm{H}->\mathrm{Asn} / \mathrm{N}$ & proto-Beijing defining & Rv2819c \\
\hline SNP & csm4 & 3127466 & $\mathrm{tcg} / \mathrm{gcg}$ & $\mathrm{Arg} / \mathrm{R}->\mathrm{Arg} / \mathrm{R}$ & BCG specific & Rv2820c \\
\hline SNP & csm4 & 3127717 & $\operatorname{tg} / \operatorname{tgc}$ & Thr/T- > Ala/A & T3_Eth defining & Rv2820c \\
\hline truncation & csm4 & 3127930 & & & Beijing RD207 truncation & Rv2820c \\
\hline SNP & $\operatorname{csm} 4$ & 3127931 & $\mathrm{ttt} / \mathrm{att}$ & Lys/K- > Asn/N & Beijing-defining & Rv2820c \\
\hline None $^{a}$ & $\operatorname{csm} 3$ & & & & & Rv2821C \\
\hline None $^{a}$ & $\operatorname{csm} 2$ & & & & & Rv2822C \\
\hline SNP & $\operatorname{cas} 10 / \mathrm{csm} 1$ & 3129359 & $\mathrm{tcc} / \mathrm{tct}$ & Gly/G- > Arg/R & CDC1551 specific & Rv2823c \\
\hline SNP & $\operatorname{cas} 10 / \mathrm{csm} 1$ & 3129675 & gcg/gtg & Arg/R- > His/H & C_0253-12 and C_0309-12 specific & Rv2823c \\
\hline SNP & $\operatorname{cas} 10 / \mathrm{csm} 1$ & 3130682 & gcc/acc & Gly/G- > Gly/G & F11 specific & Rv2823c \\
\hline SNP & $\operatorname{cas} 10 / \mathrm{csm} 1$ & 3131188 & gag/gaa & Leu/L-> Phe/F & Beijing BT2 specific & Rv2823c \\
\hline SSPS \& SNPS & $\operatorname{cas} 10 / \mathrm{csm} 1$ & 3131470 & & & Found in all MTBC strains & Rv2823c \\
\hline None $^{a}$ & cas6 & & & & & Rv2824c \\
\hline
\end{tabular}

${ }^{a}$ None: no mutations listed for the strains examined; Location: base pair of H37Rv NC_000962.3 where SNP can be found, or base pair approximately in the center of a given deletion or insertion; SSP short sequence polymorphism, SNP single nucleotide polymorphism

element is found in reverse orientation to the H37Rv mid DR IS6110 sequence [4, 41]. The results shown in this paper were consistent with these previous findings, as except for the deleted-DR Beijing strains from patient B, all Beijing mid DR IS6110 elements from validated complete chromosome sequences, had orientations inverse (Table 1, and shown in red in Additional File 6, Figure S1) to the mid DR H37Rv IS6110 orientation. In the deleted-DR Beijing strains from patient $B$, the residual mid DR IS6110 was oriented in the same direction as the reference H37Rv mid DR IS6110 mobile element (Patient B, Additional File 6: Figure S1). We speculate that generation of the deleted-DR extended RD207 deletion involved this IS6110 mobile element, which in the process was "flipped" from its previous characteristic Beijing inverse orientation (presumably generated by formation of the ancestral Beijing-defining RD207 deletion) back to a wild type H37Rv-like orientation. The mechanism behind the IS6110 ability to generate deletions has been investigated [39, 41], but to the best of our knowledge this issue has not been resolved. In summary, it is probable that the residual IS6110 mobile element found attached to the presumed (by homology) DR flanking sequences of the deleted-DR strains (Fig. 2), played an as yet uncharacterized role in the deletion of the CRISPR sequences and flanking genes observed to be deleted in the deleted-DR strains (Fig. 2).

\section{Validation of total loss of CRISPR spacers in deleted-DR isolates $A, B$, and $C$, and in downloaded read-assembled deleted-DR strain SRR1188186 Approach 1}

Recovery of spoligotypes from NGS data for isolates A, B, and C, and T3_Eth, was accomplished as follows 
(by LS, Scripps). A bed file was created using the coordinates of the 43 DR repeat units. This bedfile and the aligned bam files were used with "samtools bedcov" tool to output number of counts for each repeat unit. In order to produce a binary table matching the spoligotyping, regions with less than 500 counts were considered absent. By this method, the correct spoligotypes (spacer complement) could be obtained for the intact-DR T3_Eth.

\section{Approach 2}

In order to validate the total loss of spacers and repeats in the deleted-DR isolates, for each isolate A, B and C corrected DRplus sequences (see above: corrected by substitution of manual PCR-derived contig sequences spanning the DR region) were examined for spacer and repeat sequences, which were found to be totally absent from these corrected DRplus sequences.

\section{Approach 3}

In order to further confirm the total loss of CRISPR spacers in deleted-DR isolates $\mathrm{A}, \mathrm{B}$, and $\mathrm{C}$, and in downloaded read-assembled deleted-DR strain SRR1188186, a new application was developed (by PJF, NPHLTA) for BioNumerics 7.5 and 7.6 and Excel (manuscript in preparation). The application yielded results consistent with other approaches ( 1 and 2 above) in that no spacers were detected in deleted-DR strains, and correct spoligotypes were assigned to reference H37Rv, reference BCG, and the 3 sequenced T3_Eth.

\section{CRISPR-Cas defects: comparison of MTBC strains for insertions/deletions and SNPs in cas genes}

Since isolates from patient B were Beijing, it was of interest that the deleted-DR deletion consisted of a left-side-of-IS6110 extension (Fig. 2) of the Beijingdefining RD207 deletion. The RD207 deletion is found in all modern Beijing [15, 31], and removes most CRISPRspacers and cas genes through Rv2820 (csm4) (Fig. 2) [15]. Table 2 lists the cas gene mutations in order of ascending location on the H37Rv chromosome (although transcription is in the reverse direction), and the corresponding mutated genes for the strains examined in this study (Table 1). Notable mutations were the extensive deletions of cas genes in Beijing family members including deletion of cas1 (Fig. 2); the deletion of cas 2 and cas1 by all deleted-DR strains (Fig. 2); T3_Eth specific missense SNPs in cas1; and a proto-Beijing specific missense SNP in cas1 (Table 2, Additional file 7: Figures S4-6). T3_Eth is our largest MDR-containing African-origin cluster, and has been reported by others to have proclivity for MDR [42].
How likely is it that the Cas1 proteins from Type III-A systems in MTBC also have a role in DNA repair in a manner similar to that of Cas1 proteins from Type I-E in $E$. coli?

Babu et al. 2011 [27] induced DNA damage in E. coli by exposure to mitomycin C, UV light or cisplatin. They noted, and gave examples, that Cas1 proteins from different genera of bacteria can display related but different biochemical properties. Thus any given Cas1 protein must be characterized for its biochemical and functional roles, which they did for the E. coli Type I-E Cas1, but which has not yet been done for the MTBC Type III-A Cas1. Moreover, we and others [43] were not able to find any studies done in any genus to determine the biochemical and functional roles of Type III systems. Nevertheless, Makarova et al. 2015 [12] noted that the Cas1 family is the most conserved Cas protein family $[44,45]$ and showed that the Cas1 phylogenetic tree corresponds nicely with the phylogeny of the bacterial genera. By use of the NCBI tool CDART [46], we observed that the E. coli Type I-E Cas1 had homologous domain architecture with the M. tuberculosis Type III-A Cas1. The Cas1 proteins from both were members of the Cas1 endonuclease super-family Cas1_I-II-III. Only the E. coli Cas1 was labeled "multifunctional" because there is no relevant data yet for $M$. tuberculosis Cas1, however, we consider it likely that the MTBC Cas1 will eventually be shown to have a role in endogenous DNA repair.

It was noted by Makarova et al., 2015, [12] that the adaptation module (cas1 and cas2) is dispensable in subtypes III-A and III-B. This would tend to support the idea that Cas1 in MTBC is nonessential, even if it does have an endogenous DNA-repair activity role. Consistent with this, the Beijing family of MTBC has deleted cas1 and cas2 (Fig. 2), yet still is a very successful pathogen. However, nonessential does not necessarily mean non-important. Cas1 is produced in MTBC [30], except for the possible exceptions noted in our paper (and any other exceptions yet to be found), and its absence or mutation-induced defectiveness could in theory result in impaired DNA repair and consequent increased mutability.

\section{Has induction of the cas 1 gene been observed in response to DNA-damage in genera other than that of E. coli which shows Type I-E cas1 induction?}

Pyrococcus furiosus has 7 different CRISPR loci and 3 different CRISPR-Cas systems, Type III-B, Type I-G, and Type I-A [47]. Williams et al., 2007, [48] analyzed the transcriptome of $P$. furiosus after induction of DNA damage by gamma irradiation. They observed 5 genes that had up to a 10-fold increase in mRNA levels following gamma irradiation. 3 of the 5 genes were CRISPRassociated, but none of them was cas1, although 1 was 
predicted to be involved in DNA repair and recombination [48]. Interestingly, all 3 up-regulated CRISPRassociated genes belonged to the Type I-A system [47], and none of the genes in the other 2 systems, Type III-B and Type I-G, were similarly up-regulated [48]. Williams et al., 2007, [48] note that some efficient DNA repair pathways appear to be constitutively expressed in the hyperthermophilic archaeon $P$. furiosus, which they attribute to the result of life at high temperature. To our knowledge there are no studies of induction of cas 1 in response to DNA-damage, other than the report of induction of cas1 in E. coli [27].

Life in a macrophage, and frequent exposure to hypoxic conditions, also lead to a stressful environment for $M$. tuberculosis, and we ask if this is the reason that Cas1 was found in vitro in the MTBC proteome by Mawyuenyega et al., 2005 [30], that is, is there constitutive expression of the MTBC Type III-A cas1 gene? Support for this view comes from findings that cas1 (Rv2817c) is an essential gene for MTBC growth in vitro [49-51]. In contrast, for MTBC H37Rv grown in vivo the cas1 gene was unessential in an infected-mouse model [52] and the Cas1 protein was not detected in the proteome in an infected-guinea pig model [53]. Perhaps in vivo expression of cas genes is regulated differently than in vitro expression. In any case, if cas1 gene and Cas1 protein are essential for in vitro growth of MTBC, the deleted-DR strains and Beijing family strains (all lacking cas1, Fig. 2) and strains with potentially defective cas1 such as T3_Eth (Table 2, Additional File 7 Figures S2 and S3), must have developed compensatory mechanisms which allowed them to be cultured from clinical samples. Another possibility that has not been examined, is whether cas1 expression is somehow connected with possible constitutive expression of crRNAs - in P. furiosus the approximately 200 crRNAs from all 7 CRISPR loci are constitutively expressed (reviewed in Majumdar et al. 2015 [47]), but to our knowledge nothing is known about the expression of MTBC crRNAs.

\section{What possible effects might the loss of the cas 1 gene have on any given MTBC strain?}

Cas1 protein physically and genetically interacts with key components of $E$. coli DNA repair systems, including $\operatorname{rec} B$, $\operatorname{rec} C$ and $r u v B$, and cas1 deleted E.coli shows increased sensitivity to DNA damage and impaired chromosomal segregation [27]. Is there the possibility of a similar phenotype in the deleted-DR strains of MTBC, or intact-DR strains of MTBC, that are defective in the cas1 gene? There is no data with which to answer this question, but some pertinent observations can be made concerning MTBC. As already mentioned, the widespread successful MTBC pathogens of the Beijing family $[54,55]$ all lack the cas1 gene as part of their defining
RD207 deletion (Fig. 2, Table 2) [15, 31]. Evidence presented in this paper showed that all deleted-DR strains (assigned to Haarlem, Beijing, EAI, and CAS spoligotype families by RD deletion profiles, Table 1) lost at least their cas 1 and cas 2 genes (Fig. 2, Table 2), but previously Beijing was considered the only natural instance known of CRISPR-containing bacteria to be without a cas1 gene [27]. However this knowledge has been updated by Makarova et al., 2015, [12] who report that the cas1cas2 adaptation module is dispensable in Types III-A and III-B CRISPR-Cas systems. No evidence has been offered for impaired chromosomal segregation in Beijing MTBC, but there appears to be an increased sensitivity to DNA damage as shown by Beijing-specific SNPs resulting in a potential mutator phenotype (Table 1) [37], and by the fact that in Israel [1] and worldwide [37, 54, 56-58], MTBC Beijing family strains are observed to be robustly transmissible (at least in the patient's country of origin) and to have an increased tendency to be MDR. Thus one could speculate that in Beijing, loss of cas 1 may have contributed to increased adaptability (caused by increased mutability due to decreased DNA repair function resulting from loss of Cas1 and loss of DNA repair activity from the DNA repair genes/gene products with which Cas1 interacts $[27,59])$. In this regard, it is notable that the largest MDR-containing African-origin cluster in Israel [1] and some parts of Africa [42] is T3_Eth, which was shown in this study to have 2 potentially disruptive SNP missense mutations in its cas1 gene (Table 2, Additional file 7, Figures S2 and S3). Further studies are necessary to determine whether T3_Eth has functional Cas1.

MTBC strains differ from most other bacteria by lacking a mismatch repair system $[60,61]$, the lack of which causes instability within genomic regions containing nucleotide repeats [61]. We previously published a possible instance of this instability observed in a large cluster of CAS family MTBC in Israel [7], and one could speculate that this instability may be one of the drivers of MIRU-VNTR polymorphism in general which is extremely useful in molecular epidemiology of MTBC [5]. However, Wanner et al. [61] analyzed the MTBC strain $\mathrm{H} 37 \mathrm{Rv}$ and other bacteria, and determined that deficiency in DNA-repair increases the selection for inherently stable genomes "by using codons to encode proteins in a context-dependent manner that prevents the emergence of nucleotide repeats." The results reported and summarized in our paper suggest that different families of MTBC have different burdens of DNA-repair deficiency (Table 1, Table 2), and thus the important observation of stabilization of the $\mathrm{H} 37 \mathrm{Rv}$ genome may not be generalizable to all MTBC without examining other strains belonging to at least the other major families of MTBC. 
The effect of defective DNA-repair genes is cumulative: the more defective DNA-repair genes, the more mutability [62], which presumably could lead to increased adaptability, with consequent accumulation of mutations in global transcription/stress response genes favoring survivability/ persistence, transmissibility, and acquisition of drug resistance. All MTBC strains, as noted above, lack the mismatch repair system, and it is therefore important to draw attention to the possible cumulative effect in Beijing set in motion by the additional loss of cas1 (Fig. 2, Table 2) and its putative associated DNA-repair network [27], in addition to other known Beijing-specific defective DNA-repair genes, (Table 1, [37]). The DNA-repair mechanism in MTBC is already known to be one of the slowest in bacteria [59], and cumulative loss of DNArepair functionality could only be expected to make the DNA-repair mechanism even slower, exacerbating the acquisition of mutations known to result from defective DNA-repair [62].

\section{Conclusions}

Deleted-DR strains showed complete loss of clustered regularly interspaced short palindromic repeats (CRISPR) and accompanying spacers, retention of the mid-CRISPR IS6110 mobile element in clonally specific orientation, and accompanying clonally-specific loss of flanking genes, including adjacent CRISPR-associated (cas) genes [12]. All deleted-DR strains were accompanied by a loss of at least the cas 2 and cas 1 genes. Tentative MIRU-VNTR-based assignments of the deleted-DR isolates to MTBC major spoligotype families A Haarlem, B Beijing, and C EAI were validated by identifying lineage-defining long sequence polymorphisms (LSPs) known as region of difference (RD) deletions $[14,15,17]$, corresponding to major spoligotype families [17]. Similarly, the deleted-DR strain SRR1188186 whose sequence was assembled from downloaded reads, deleted cas 2 and cas 1 and was assigned by RD deletion to the MTBC major spoligotype family CAS. Since cas 1 deleted E.coli shows increased sensitivity to DNA damage and impaired chromosomal segregation [27], we discussed the possibility of a similar phenotype in the deleted-DR strains of MTBC and in the intact-DR MTBC Beijing that are defective in the cas1 gene (having deleted it), and in the intact-DR MTBC T3_Eth that are potentially defective in the cas 1 gene (having 2 potentially disruptive missense SNPs in it).

\section{Additional files}

Additional file 1: Table S1. MIRU-VNTR loci and octal spoligotypes Samples: A-B-C\&T3_Eth. Controls: 0486-12 Beijing clinical isolate, ECDCPT 21\&25 EAl, reference H37Rv, Pasteur BCGP2. (DOCX 19 kb)

Additional file 2: List S1. Sources for drug sensitivity data presented in Table 1. (DOCX $15 \mathrm{~kb})$
Additional file 3: Sequences S1-S6. (ZIP $66 \mathrm{~kb})$

Additional file 4: Table S2. Location of CRISPR-flanking genes and mid-CRISPR IS6110 mobile element used for primer choices and alignments. (DOCX $17 \mathrm{~kb}$ )

Additional file 5: Table S3. Primers employed for PCR verification of deletions of CRISPR/DR and flanking regions. (DOCX $14 \mathrm{~kb}$ )

Additional file 6: Figure S1. Visualization of mid IS6110 orientation: blue for wild type (as in reference H37Rv) or for unknown orientation; red for inverse orientation (see Table 1). DR plus flanking sequences were aligned to DR and flanking regions of reference template H37Rv. Sequences were from Mycobacterium tuberculosis NGS read sets generated in this study (patients $A, B$, and C corrected by manual PCR; and T3_Eth), on-line resources of read sets, mapped on H37Rv

NC_000962.3, and on-line resources of complete chromosome sequences. (PPT $481 \mathrm{~kb}$ )

Additional file 7: Figures S2-S4. Mutations of cas1 shared by MTBC T3_Eth strains and by Proto-Beijing strains. (PPTX 2956 kb)

\section{Abbreviations}

Cas: CRISPR-associated; CAS: Central Asian (refers to a major spoligotype and corresponding RD deletion family); cas: CRISPR-associated gene; CRISPR: Clustered regularly interspaced short palindromic repeats; DR: Direct repeat region; DRplus: Sequence of DR plus flanking regions - about $40 \mathrm{Kbp}$; LSP: Long sequence polymorphism; MIRU-VNTR: Mycobacterial interspersed repetitive units - variable number tandem repeats; MTBC: Mycobacterium tuberculosis complex; NGS: Next generation sequencing; RD: Region of difference; spoligotyping: Spacer oligonucleotide typing

\section{Acknowledgments}

We thank the staff of the National Mycobacterium Reference Center for routine excellent performance of Mycobacterium tuberculosis growth, identification and drug sensitivity tests, and maintenance of the Mycobacterium tuberculosis strain repository.

\section{Funding}

This research did not receive any specific grant from funding agencies in the public, commercial, or not-for-profit sectors.

\section{Availability of data and materials}

NGS sequence reads for the 6 deleted-DR MTBC isolates from patients A Haarlem, B Beijing, and C EAI and for 2 intact-DR MTBC T3_Eth, have been deposited in the international DNA database GenBank under BioProject PRJNA309637, accession no. SRP068918. All other datasets supporting the results of this article are available in the main paper and additional files, or by downloading from international databases such as GenBank.

\section{Authors' contributions}

PJF, IN, DG, and ER conceived and designed the study. PJF, IN, AL, SRH, and LS conducted the molecular work and data analysis. LS constructed a method to obtain spoligotypes from NGS reads. PJF constructed an application employing BioNumerics (Applied Maths, Belgium) and Excel (Microsoft Company) to obtain spoligotypes from NGS reads and complete whole genome sequences. PJF, IN, $D G$, and ER drafted the manuscript. PJF, DG, and ER coordinated the study. ER, HK, ZD and DC contributed to the development of the project. All authors have read and approved the final manuscript.

\section{Competing interests}

The authors declare that they have no competing interests.

\section{Consent for publication}

Not applicable.

\section{Ethics approval and consent to participate}

No ethics approval was required for the public sequence data used in this study. No ethics approval was required for the anonymous use of Mycobacterium tuberculosis strains stored in the Mycobacterium tuberculosis Repository of the National Mycobacterium Reference Center. 


\section{Agreement to be a co-author}

All authors have agreed to be co-authors of this article: "Structure and variation of CRISPR and CRISPR-flanking regions in deleted-direct repeat region Mycobacterium tuberculosis complex strains".

\section{Author details}

${ }^{1}$ National Mycobacterium Reference Center, National Public Health Laboratory Tel Aviv, Ministry of Health, Tel Aviv, Israel. ${ }^{2}$ Current address: Ben-Gurion University of the Negev, Beer Sheva, Israel. ${ }^{3}$ Scripps Research Institute, San Diego, CA, USA. ${ }^{4}$ Department of Tuberculosis and AIDS, Ministry of Health, Jerusalem, Israel. ${ }^{5}$ National Public Health Laboratory Tel Aviv, Ministry of Health, Tel Aviv, Israel.

\section{Received: 10 October 2016 Accepted: 7 February 2017} Published online: 15 February 2017

\section{References}

1. Goldblatt D, Rorman E, Chemtob D, Freidlin PJ, Cedar N, Kaidar-Shwartz H, Dveyrin Z, Mor Z. Molecular epidemiology and mapping of tuberculosis in Israel: do migrants transmit the disease to locals? Int J Tuberc Lung Dis. 2014;18(9):1085-91.

2. Dale JW, Brittain D, Cataldi AA, Cousins D, Crawford JT, Driscoll J, Heersma H, Lillebaek T, Quitugua T, Rastogi N, et al. Spacer oligonucleotide typing of bacteria of the Mycobacterium tuberculosis complex: recommendations for standardised nomenclature. Int J Tuberc Lung Dis. 2001;5(3):216-9.

3. Kamerbeek J, Schouls L, Kolk A, van Agterveld M, van Soolingen D, Kuijper S, Bunschoten A, Molhuizen H, Shaw R, Goyal M, et al. Simultaneous detection and strain differentiation of Mycobacterium tuberculosis for diagnosis and epidemiology. J Clin Microbiol. 1997;35(4):907-14.

4. van Embden JD, van Gorkom T, Kremer K, Jansen R, van Der Zeijst BA, Schouls LM. Genetic variation and evolutionary origin of the direct repeat locus of Mycobacterium tuberculosis complex bacteria. J Bacteriol. 2000;182(9):2393-401.

5. Supply P, Allix C, Lesjean S, Cardoso-Oelemann M, Rusch-Gerdes S, Willery E, Savine E, de Haas P, van Deutekom H, Roring S, et al. Proposal for standardization of optimized mycobacterial interspersed repetitive unitvariable-number tandem repeat typing of Mycobacterium tuberculosis. J Clin Microbiol. 2006;44(12):4498-510.

6. Freidlin P, Goldblatt D, Kaidar-Shwartz H, Dveyrin Z, Rorman E. Quality assurance for molecular epidemiology of tuberculosis methods in the mycobacterium reference laboratory. Accred Qual Assur. 2011;16(12):623-35.

7. Freidlin PJ, Goldblatt D, Kaidar-Shwartz H, Rorman E. Polymorphic exact tandem repeat A (PETRA): a newly defined lineage of mycobacterium tuberculosis in israel originating predominantly in Sub-Saharan Africa. J Clin Microbiol. 2009;47(12):4006-20.

8. Cabal A, Strunk M, Dominguez J, Lezcano MA, Vitoria MA, Ferrero M, Martin C, Iglesias MJ, Samper S. Single nucleotide polymorphism (SNP) analysis used for the phylogeny of the Mycobacterium tuberculosis complex based on a pyrosequencing assay. BMC Microbiol. 2014;14:21.

9. Parsons SD, Drewe JA, van Gey Pittius NC, Warren RM, van Helden PD. Novel cause of tuberculosis in meerkats, South Africa. Emerg Infect Dis. 2013;19(12):2004-7.

10. Demay C, Liens B, Burguiere T, Hill V, Couvin D, Millet J, Mokrousov I, Sola C, Zozio T, Rastogi N. SITVITWEB-a publicly available international multimarker database for studying Mycobacterium tuberculosis genetic diversity and molecular epidemiology. Infect Genet Evol. 2012;12(4):755-66.

11. Zegans ME, Wagner JC, Cady KC, Murphy DM, Hammond JH, O'Toole GA. Interaction between bacteriophage DMS3 and host CRISPR region inhibits group behaviors of Pseudomonas aeruginosa. J Bacteriol. 2009;191(1):210-9.

12. Makarova KS, Wolf YI, Alkhnbashi OS, Costa F, Shah SA, Saunders SJ, Barrangou R, Brouns SJ, Charpentier E, Haft DH, et al. An updated evolutionary classification of CRISPR-Cas systems. Nat Rev Microbiol. 2015;13(11):722-36.

13. Caimi K, Cataldi A. A fragment of 21 ORFs around the direct repeat (DR) region of Mycobacterium tuberculosis is absent from the other sequenced mycobacterial genomes: implications for the evolution of the DR region. Comp Funct Genomics. 2004;5(2):116-22.

14. Tsolaki AG, Hirsh AE, DeRiemer K, Enciso JA, Wong MZ, Hannan M, Goguet de la Salmoniere YO, Aman K, Kato-Maeda M, Small PM. Functional and evolutionary genomics of Mycobacterium tuberculosis: insights from genomic deletions in 100 strains. Proc Natl Acad Sci U S A. 2004;101(14):4865-70.
15. Gagneux S, DeRiemer K, Van T, Kato-Maeda M, de Jong BC, Narayanan S, Nicol M, Niemann S, Kremer K, Gutierrez MC, et al. Variable host-pathogen compatibility in Mycobacterium tuberculosis. Proc Natl Acad Sci U S A. 2006;103(8):2869-73.

16. Cole ST, Brosch R, Parkhill J, Garnier T, Churcher C, Harris D, Gordon SV, Eiglmeier K, Gas S, Barry 3rd CE, et al. Deciphering the biology of Mycobacterium tuberculosis from the complete genome sequence. Nature. 1998;393(6685):537-44.

17. Coll F, McNerney R, Guerra-Assuncao JA, Glynn JR, Perdigao J, Viveiros M, Portugal I, Pain A, Martin N, Clark TG. A robust SNP barcode for typing Mycobacterium tuberculosis complex strains. Nat Commun. 2014;5:4812.

18. Brudey K, Driscoll JR, Rigouts L, Prodinger WM, Gori A, Al-Hajoj SA, Allix C, Aristimuno L, Arora J, Baumanis $V$, et al. Mycobacterium tuberculosis complex genetic diversity: mining the fourth international spoligotyping database (SpolDB4) for classification, population genetics and epidemiology. BMC Microbiol. 2006;6:23.

19. Coll F, Mallard K, Preston MD, Bentley S, Parkhill J, McNerney R, Martin N, Clark TG. SpolPred: rapid and accurate prediction of Mycobacterium tuberculosis spoligotypes from short genomic sequences. Bioinformatics. 2012;28(22):2991-3.

20. Sekizuka T, Yamashita A, Murase Y, Iwamoto T, Mitarai S, Kato S, Kuroda M. TGS-TB: Total Genotyping Solution for Mycobacterium tuberculosis Using Short-Read Whole-Genome Sequencing. PLoS One. 2015;10(11):e0142951.

21. Tang C, Reyes JF, Luciani F, Francis AR, Tanaka MM. spolTools: online utilities for analyzing spoligotypes of the Mycobacterium tuberculosis complex. Bioinformatics. 2008;24(20):2414-5.

22. Xia E, Teo YY, Ong RT. SpoTyping: fast and accurate in silico Mycobacterium spoligotyping from sequence reads. Genome Med. 2016;8(1):19.

23. Niemann S, Koser CU, Gagneux S, Plinke C, Homolka S, Bignell H, Carter RJ, Cheetham RK, Cox A, Gormley NA, et al. Genomic diversity among drug sensitive and multidrug resistant isolates of Mycobacterium tuberculosis with identical DNA fingerprints. PLoS One. 2009;4(10):e7407.

24. Walker TM, Ip CL, Harrell RH, Evans JT, Kapatai G, Dedicoat MJ, Eyre DW, Wilson DJ, Hawkey PM, Crook DW, et al. Whole-genome sequencing to delineate Mycobacterium tuberculosis outbreaks: a retrospective observational study. Lancet Infect Dis. 2013;13(2):137-46.

25. Garneau JE, Dupuis ME, Villion M, Romero DA, Barrangou R, Boyaval P, Fremaux C, Horvath P, Magadan AH, Moineau S. The CRISPR/Cas bacterial immune system cleaves bacteriophage and plasmid DNA. Nature. 2010;468(7320):67-71.

26. Marraffini LA, Sontheimer EJ. CRISPR interference limits horizontal gene transfer in staphylococci by targeting DNA. Science. 2008;322(5909):1843-5.

27. Babu M, Beloglazova N, Flick R, Graham C, Skarina T, Nocek B, Gagarinova A, Pogoutse O, Brown G, Binkowski A, et al. A dual function of the CRISPR-Cas system in bacterial antivirus immunity and DNA repair. Mol Microbiol. 2011;79(2):484-502.

28. Fenner L, Malla B, Ninet B, Dubuis O, Stucki D, Borrell S, Huna T, Bodmer T, Egger M, Gagneux S. "Pseudo-Beijing": evidence for convergent evolution in the direct repeat region of Mycobacterium tuberculosis. PLoS One. 2011;6(9):e24737.

29. He L, Fan X, Xie J. Comparative genomic structures of Mycobacterium CRISPR-Cas. J Cell Biochem. 2012;113(7):2464-73.

30. Mawuenyega KG, Forst CV, Dobos KM, Belisle JT, Chen J, Bradbury EM, Bradbury AR, Chen X. Mycobacterium tuberculosis functional network analysis by global subcellular protein profiling. Mol Biol Cell. 2005;16(1):396-404.

31. Luo T, Comas I, Luo D, Lu B, Wu J, Wei L, Yang C, Liu Q, Gan M, Sun G, et al. Southern East Asian origin and coexpansion of Mycobacterium tuberculosis Beijing family with Han Chinese. Proc Natl Acad Sci U S A. 2015;112(26):8136-41.

32. Huang Q, Luo H, Liu M, Zeng J, Abdalla AE, Duan X, Li Q, Xie J. The effect of Mycobacterium tuberculosis CRISPR-associated Cas2 (Rv2816c) on stress response genes expression, morphology and macrophage survival of Mycobacterium smegmatis. Infect Genet Evol. 2016;40:295-301.

33. Beloglazova N, Brown G, Zimmerman MD, Proudfoot M, Makarova KS, Kudritska M, Kochinyan S, Wang S, Chruszcz M, Minor W, et al. A novel family of sequence-specific endoribonucleases associated with the clustered regularly interspaced short palindromic repeats. J Biol Chem. 2008;283(29):20361-71.

34. de Beer JL, Kodmon C, van Ingen J, Supply P, van Soolingen D, Global Network for Molecular Surveillance of T. Second worldwide proficiency study on variable number of tandem repeats typing of Mycobacterium tuberculosis complex. Int J Tuberc Lung Dis. 2014;18(5):594-600. 
35. Jansen R, Embden JD, Gaastra W, Schouls LM. Identification of genes that are associated with DNA repeats in prokaryotes. Mol Microbiol. 2002;43(6):1565-75.

36. Coll F, McNerney R, Preston MD, Guerra-Assuncao JA, Warry A, Hill-Cawthorne G, Mallard K, Nair M, Miranda A, Alves A, et al. Rapid determination of antituberculosis drug resistance from whole-genome sequences. Genome Med. 2015;7(1):51

37. Ebrahimi-Rad M, Bifani P, Martin C, Kremer K, Samper S, Rauzier J, Kreiswirth B, Blazquez J, Jouan $M$, van Soolingen $D$, et al. Mutations in putative mutator genes of Mycobacterium tuberculosis strains of the W-Beijing family. Emerg Infect Dis. 2003;9(7):838-45.

38. Roychowdhury T, Mandal S, Bhattacharya A. Analysis of IS6110 insertion sites provide a glimpse into genome evolution of Mycobacterium tuberculosis. Sci Rep. 2015;5:12567.

39. Fang Z, Morrison N, Watt B, Doig C, Forbes KJ. IS6110 transposition and evolutionary scenario of the direct repeat locus in a group of closely related Mycobacterium tuberculosis strains. J Bacteriol. 1998;180(8):2102-9.

40. Legrand E, Filliol I, Sola C, Rastogi N. Use of spoligotyping to study the evolution of the direct repeat locus by IS6110 transposition in Mycobacterium tuberculosis. J Clin Microbiol. 2001;39(4):1595-9.

41. Sampson SL, Warren RM, Richardson M, Victor TC, Jordaan AM, van der Spuy GD, van Helden PD. IS6110-mediated deletion polymorphism in the direct repeat region of clinical isolates of Mycobacterium tuberculosis. J Bacteriol. 2003;185(9):2856-66.

42. Agonafir M, Lemma E, Wolde-Meskel D, Goshu S, Santhanam A, Girmachew F, Demissie D, Getahun M, Gebeyehu M, van Soolingen D. Phenotypic and genotypic analysis of multidrug-resistant tuberculosis in Ethiopia. Int J Tuberc Lung Dis. 2010;14(10):1259-65.

43. Amitai G, Sorek R. CRISPR-Cas adaptation: insights into the mechanism of action. Nat Rev Microbiol. 2016;14(2):67-76.

44. Makarova KS, Haft DH, Barrangou R, Brouns SJ, Charpentier E, Horvath P, Moineau S, Mojica FJ, Wolf YI, Yakunin AF, et al. Evolution and classification of the CRISPR-Cas systems. Nat Rev Microbiol. 2011;9(6):467-77.

45. Takeuchi N, Wolf Yl, Makarova KS, Koonin EV. Nature and intensity of selection pressure on CRISPR-associated genes. J Bacteriol. 2012;194(5):1216-25.

46. Geer LY, Domrachev M, Lipman DJ, Bryant SH. CDART: protein homology by domain architecture. Genome Res. 2002;12(10):1619-23.

47. Majumdar S, Zhao P, Pfister NT, Compton M, Olson S, Glover 3rd CV, Wells L, Graveley BR, Terns RM, Terns MP. Three CRISPR-Cas immune effector complexes coexist in Pyrococcus furiosus. RNA. 2015;21(6):1147-58.

48. Williams E, Lowe TM, Savas J, DiRuggiero J. Microarray analysis of the hyperthermophilic archaeon Pyrococcus furiosus exposed to gamma irradiation. Extremophiles. 2007;11(1):19-29.

49. Griffin JE, Gawronski JD, Dejesus MA, loerger TR, Akerley BJ, Sassetti CM. High-resolution phenotypic profiling defines genes essential for mycobacterial growth and cholesterol catabolism. PLoS Pathog. 2011;7(9):e1002251

50. Sassetti CM, Boyd DH, Rubin EJ. Genes required for mycobacterial growth defined by high density mutagenesis. Mol Microbiol. 2003;48(1):77-84

51. Zhang YJ, loerger TR, Huttenhower C, Long JE, Sassetti CM, Sacchettini JC, Rubin EJ. Global assessment of genomic regions required for growth in Mycobacterium tuberculosis. PLoS Pathog. 2012;8(9):e1002946.

52. Sassetti CM, Rubin EJ. Genetic requirements for mycobacterial survival during infection. Proc Natl Acad Sci U S A. 2003;100(22):12989-94.

53. Kruh NA, Troudt J, Izzo A, Prenni J, Dobos KM. Portrait of a pathogen: the Mycobacterium tuberculosis proteome in vivo. PLoS One. 2010;5(11):e13938.

54. Bifani PJ, Mathema B, Kurepina NE, Kreiswirth BN. Global dissemination of the Mycobacterium tuberculosis W-Beijing family strains. Trends Microbiol. 2002;10(1):45-52.

55. Filliol I, Driscoll JR, Van Soolingen D, Kreiswirth BN, Kremer K, Valetudie G, Anh DD, Barlow R, Banerjee D, Bifani PJ, et al. Global distribution of Mycobacterium tuberculosis spoligotypes. Emerg Infect Dis. 2002:8(11):1347-9.

56. Tsolaki AG, Gagneux S, Pym AS, Goguet de la Salmoniere YO, Kreiswirth BN, Van Soolingen D, Small PM. Genomic deletions classify the Beijing/W strains as a distinct genetic lineage of Mycobacterium tuberculosis. J Clin Microbiol. 2005;43(7):3185-91.

57. Schurch AC, Kremer K, Hendriks AC, Freyee B, McEvoy CR, van Crevel R, Boeree MJ, van Helden P, Warren RM, Siezen RJ, et al. SNP/RD typing of Mycobacterium tuberculosis Beijing strains reveals local and worldwide disseminated clonal complexes. PLoS One. 2011;6(12):e28365.
58. Mestre O, Luo T, Dos Vultos T, Kremer K, Murray A, Namouchi A, Jackson C, Rauzier J, Bifani P, Warren R, et al. Phylogeny of Mycobacterium tuberculosis Beijing strains constructed from polymorphisms in genes involved in DNA replication, recombination and repair. PLoS One. 2011;6(1):e16020.

59. Chaturvedi I, Rajapakse JC. Detecting robust time-delayed regulation in Mycobacterium tuberculosis. BMC Genomics. 2009;10 Suppl 3:S28.

60. Springer B, Sander P, Sedlacek L, Hardt WD, Mizrahi V, Schar P, Bottger EC. Lack of mismatch correction facilitates genome evolution in mycobacteria. Mol Microbiol. 2004:53(6):1601-9.

61. Wanner RM, Guthlein C, Springer B, Bottger EC, Ackermann M. Stabilization of the genome of the mismatch repair deficient Mycobacterium tuberculosis by context-dependent codon choice. BMC Genomics. 2008:9:249.

62. Selifonova O, Valle F, Schellenberger V. Rapid evolution of novel traits in microorganisms. Appl Environ Microbiol. 2001;67(8):3645-9.

\section{Submit your next manuscript to BioMed Central and we will help you at every step:}

- We accept pre-submission inquiries

- Our selector tool helps you to find the most relevant journal

- We provide round the clock customer support

- Convenient online submission

- Thorough peer review

- Inclusion in PubMed and all major indexing services

- Maximum visibility for your research

Submit your manuscript at www.biomedcentral.com/submit
) Biomed Central 